\title{
Comment on ' 30 years follow-up and increased risks of breast cancer and leukaemia after long-term low-dose-rate radiation exposure'
} Mohan Doss*,1

\author{
${ }^{1}$ Diagnostic Imaging, Fox Chase Cancer Center, 333 Cottman Avenue, Philadelphia, PA 19111, USA
}

Sir,

This letter is regarding the article by Hsieh et al (2017) entitled ' 30 years follow-up and increased risks of breast cancer and leukaemia after long-term low-dose-rate radiation exposure' published in British Journal of Cancer. This article is a recent update to the cancer risks in residents of the Co-60 contaminated buildings in Taiwan that was reported in 2006 (Hwang et al, 2006) and updated in 2008 (Hwang et al, 2008). Hsieh et al used Cox proportional hazard models to determine the hazard ratios for cancer incidence in the irradiated residents, and claimed that dose-dependent risks were statistically significant for leukaemia excluding chronic lymphocytic leukaemia, breast cancers, and all cancers. They also claimed that radiation exposure before age 20 was associated with a significantly increased risk of breast cancer. These conclusions are similar to the conclusions of the 2008 update (Hwang et al, 2008). On the other hand, in the 2006 report, which compared cancer rates of the irradiated population with the cancer rates of an equivalent control population, 95 cancer cases were observed up to the end of 2002 in contrast to 114.9 expected (Hwang et al, 2006), resulting in standardised incidence ratio (SIR) for all cancers of 0.83 (95\% CI: 0.66-0.99), indicating a significant reduction of all cancers following low-dose irradiation. Let us now examine whether SIR in the recently updated data shows a significant reduction of all cancers.

The Hsieh et al publication reports that 249 cancer cases were observed in the cohort up to the end of 2012. To calculate the SIR, we need to know the expected number of cancer cases for the same period. In the 2006 report, Hwang et al reported that the expected number of all cancers was 114.9 , and the average age of the irradiated cohort was 33.3 at the end of 2002 (The average age of the population was 17.1 at the time of irradiation and the cohort was followed-up for an average of 16.2 years) (Hwang et al, 2006). Hence, for the Hsieh et al publication, the average age at the end of the study period (end of 2012) would be 43.3. The cancer incidence rates for the ages of 33.3 and 43.3, obtained by interpolation of the average of male and female cancer incidence rates during 1998-2002 from Taiwan Cancer Registry (TCR, 2008), are 86.3 and 222.4, respectively, indicating there would be an increase in cancer incidence between these two ages by a factor of $\sim 2.58$. Therefore, considering the 114.9 expected cases to the end of 2002 (Hwang et al, 2006), the expected cancer cases up to the end of 2012 would be 296.4, resulting in a SIR of $249 / 296.4=0.84$ (95\% CI: $0.74-0.95)$. Thus, the reduction of cancer rate in the irradiated cohort is significant in the updated data also. A similar analysis of the data published in 2008 (Hwang et al, 2008) shows that SIR for that study would be 0.75 (95\% CI: $0.61-0.88$ ), based on 117 observed and 156.8 expected cancers to the end of 2005, again indicating reduction of all cancers in the irradiated cohort. Hsieh et al have failed to discuss the significant reduction of overall cancers in the irradiated cohort.

When there is a significant reduction of all cancers, a few of the individual cancers may show increased incidence because of statistical fluctuations, and the increase may indeed reach statistical significance, especially when $90 \%$ CIs are used. This should not be interpreted as increased risk for these cancer types, but additional data with higher statistics should be obtained before reaching any conclusion.

One major problem with the use of the proportional hazard models for estimating hazard ratios is that the results from such analysis, for example, the results reported by Hsieh et al, can mask the reduction of cancers that is observed in the irradiated cohort. When there is observed reduction of all cancers following low-dose-radiation exposures, use of the proportional hazard model for estimating the hazard from low radiation doses is not justified and it would result in erroneous conclusions.

In summary, the conclusion of the Hsieh et al publication that protracted, low-dose-rate radiation exposures increase cancer risk for certain types of cancers and for younger age groups is not valid, because of their use of proportional risk model to calculate hazard ratios when significant reduction of all cancers has been observed following low-doseradiation exposures. On the other hand, a consistent conclusion from the analysis of the data published over the years on the Taiwan irradiated cohort (Hwang et al, 2006, 2008; Hsieh et al, 2017) is that the risk of all cancers is significantly reduced, in comparison to an equivalent control population.

\section{CONFLICT OF INTEREST}

The author declares no conflict of interest.

\section{REFERENCES}

Hsieh WH, Lin IF, Ho JC, Chang PW (2017) 30 years follow-up and increased risks of breast cancer and leukaemia after long-term low-dose-rate radiation exposure. Br J Cancer 117: 1883-1887.

Hwang SL, Guo HR, Hsieh WA, Hwang JS, Lee SD, Tang JL, Chen CC, Chang TC, Wang JD, Chang WP (2006) Cancer risks in a population with prolonged low dose-rate gamma-radiation exposure in radiocontaminated buildings, 19832002. Int J Radiat Biol 82: 849-858.

Hwang SL, Hwang JS, Yang YT, Hsieh WA, Chang TC, Guo HR, Tsai MH, Tang JL, Lin IF, Chang WP (2008) Estimates of relative risks for cancers in a population after prolonged low-dose-rate radiation exposure: a follow-up assessment from 1983 to 2005. Radiation Res 170: 143-148.

TCR (2008) Cancer Incidence Rate in Taiwan, 1998-2002 \& 2003-2007. Taiwan Cancer Registry. Available at: http://tcr.cph.ntu.edu.tw/main.php?Page=N2.

This work is published under the standard license to publish agreement. After 12 months the work will become freely available and the license terms will switch to a Creative Commons Attribution-NonCommercial-Share Alike 4.0 Unported License. 